\title{
Characterisation of Epitaxial Lateral Overgrown GaN by Electron Backscatter Diffraction Correlated with Cross-Sectional Cathodoluminescence Spectroscopy
}

\author{
F. Sweeney, ${ }^{*}$ C. Trager-Cowan, ${ }^{*}$ P. R. Edwards, ${ }^{*}$ A. J. Wilkinson, ${ }^{* *}$ and I. M. Watson*** \\ * SUPA, Department of Physics, University of Strathclyde, Glasgow G4 ONG, Scotland, UK \\ ** Department of Materials, University of Oxford, Oxford OX1 3PH, UK \\ *** SUPA, Institute of Photonics, University of Strathclyde, Glasgow G4 ONW, Scotland, UK
}

Nitride semiconductor based light emitting diodes (LEDs) and laser diodes (LDs) are now being extensively used in consumer electronics because they are durable, energy efficient and available with bright light emission from the Ultra-Violet to the green region of the spectrum. These nitridebased optoelectronic devices are now being employed in traffic lights, backlights for displays, BlueRay DVD, surgeon's goggles and curing lamps for dentistry (Fig. 1). However the most exciting development in the nitrides has been the advent of the white nitride based LED. These white LEDs are set to replace traditional incandescent and fluorescent lighting and are therefore going to revolutionise the way our homes and offices are illuminated in the next decade.

Manufacturers of nitride based LEDs and LDs are seeking ways to increase device efficiency and longevity. To achieve this dislocation densities in nitride films have to be reduced; typically a Gallium Nitride $(\mathrm{GaN})$ epilayer grown heteroepitaxially on a sapphire substrate can have a dislocation density as high as $10^{10} \mathrm{~cm}^{-2}$. A common growth method for reducing dislocation densities in GaN is Epitaxial Lateral Overgrowth (ELO). In the ELO growth process the final epilayer grows vertically and laterally simultaneously through and over a dielectric mask. This can results in a $10^{3}$ reduction in the dislocation density in the ELO regions above the mask.

In this presentation we shall report on our strain investigations of a just-coalesced $4 \mu \mathrm{m}$ thick ELO GaN epitaxial layer. In our study we used two independent techniques: Cathodoluminescence (CL) hyper-spectral imaging and Electron Backscatter Diffraction (EBSD). CL allows us to attain a wealth of information about the optical properties of a crystalline semiconductor with sub-micron resolution. The width and position of a peak in a luminescence spectrum is sensitive to strain, crystallinity, defects, doping and free carrier concentrations. EBSD is a technique for probing the structural properties with high spatial resolution $(\sim 50 \mathrm{~nm})$. An EBSD pattern is a direct 2-D gnomonic projection of the crystal structure and is therefore suitable for the observation of changes in the crystal structure due to strains, tilts and rotations. The EBSD strain resolution achieved in this work is $\pm 2 \times 10^{-4}[1]$.
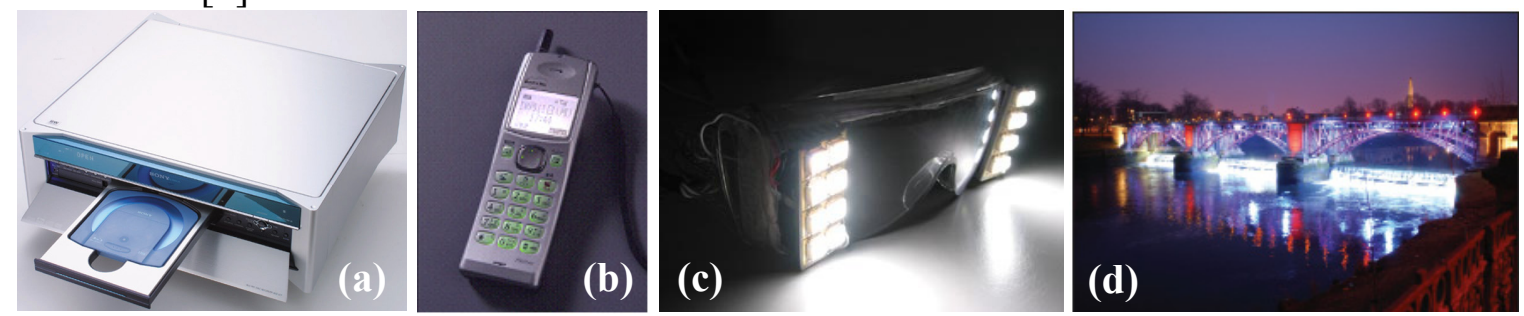

Fig 1: Applications of nitride semiconductors (a) World's first ever blue-ray DVD recorder (b) LED Backlit Mobile Phone (c) White LED goggles for surgeons and (d) "The Weir" in the City of Glasgow floodlit with nitride LED's, courtesy of Steve Hosey. 
EBSD strain profiles reveal a difference of approximately $6 \times 10^{-3}\left(3 \times 10^{-3}\right)$ in the a-axis (caxis) or in-plane (out of plane) compressive (tensile) strain between the seed-wing interface and the wing-wing coalescence boundary, see Fig. 2a. Using these differences a strain shift of $\sim 50 \mathrm{meV}$ in the CL near bandedge peak (NBEP) position is predicted [2].

CL hyper-spectral imaging of the ELO sample was carried out in cross-section. In a CL spectrum the NBEP position is affected by strain and by carrier concentration. The CL data revealed that the NBEP changed both in position and width (see Fig. 2b) between the seed-wing interface and the wing-wing coalescence boundary by $\sim 15 \mathrm{meV}$ and $\sim 38$ $\mathrm{meV}$, respectively.

Previous reports show that changes in free carrier concentration in $\mathrm{GaN}$ between approximately $10^{16} \mathrm{~cm}^{-2}$ and $10^{20} \mathrm{~cm}^{-2}$ results in an increase in the NBEP width and a red-shift (to longer wavelengths) in the NBEP position [3-4]. From the $38 \mathrm{meV}$ increase in CL peak width observed between the seed-wing interface and the wing-wing coalescence boundary, we estimate using experimentally derived models, that the carrier concentration varies by three orders of magnitude and the resulting red shift in the NBEP due to carrier concentration variation is $\sim 33 \mathrm{meV}$. Using this value to derive the shift of the CL peak due to strain alone gives a strain shift of 48 $\mathrm{meV}$. This is in very good agreement with the EBSD predicted shift of $50 \mathrm{meV}$ and confirms the validity of our EBSD strain results (see Fig 2c).

This investigation has demonstrated that by using a multi-technique approach the effects of carrier concentration and strain on the luminescence properties can be deconvoluted.

\section{References}

[1] A. J. Wilkinson, J. of Electron Microscopy 49 (2) 299-310 (2000)

[2] W. Shan et al, Phys. Rev. B. 54 (19) 13460 (1996)

[3] I-H Lee et al, APL 74 (1) 102 (1999)

[4] M. Yoshikawa, APL 86 (8) 4400 (1999)
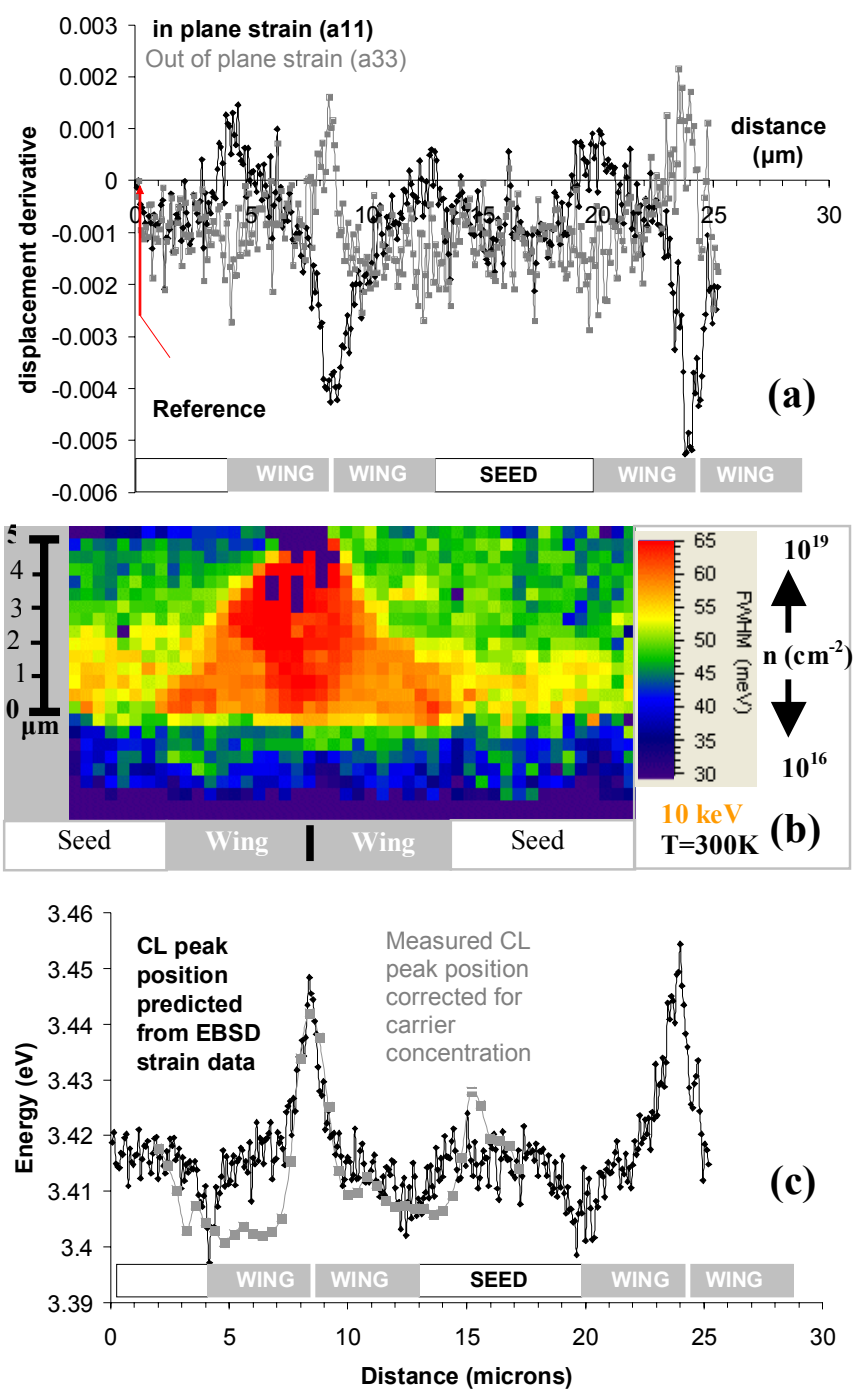

Fig. 2: (a) EBSD in-plane and out of plane strain profiles (b) CL cross-sectional peak width map and (c) Superposition of EBSD predicted CL peak position and the carrier concentration corrected CL peak position 